\title{
Relationship Between Immunological Factors and Hemarthrosis in Hemophiliacs in Antananarivo Madagascar
}

\author{
Rakotomalala Toky Randriamahazo ${ }^{1, *}$, Zoliarisoa Ramihajamanana ${ }^{2}$, \\ Anjatiana Annick Raherinaivo ${ }^{1}$, Miora Rasamindrakotroka ${ }^{1}$, Davidra Rajaonatahina ${ }^{1}$, \\ Olivat Rakoto Alson ${ }^{2}$, Andry Rasamindrakotroka ${ }^{1}$ \\ ${ }^{1}$ Laboratory of Immunology, Joseph Ravoahangy Andrianavalona University Hospital Center (JRA UHC) Antananarivo, Madagascar \\ ${ }^{2}$ Laboratory of Hematology, Joseph Ravoahangy Andrianavalona University Hospital Center (JRA UHC) Antananarivo, Madagascar
}

\section{Email address:}

drrtoky@yahoo.fr (R. T. Randriamahazo), azoliharisoa@gmail.com (Z. Ramihajamanana), anjatianaannick@yahoo.fr (A. A. Raherinaivo), mioratantely31@yahoo.fr (M. Rasamindrakotroka),rdavidra@yahoo.fr (D. Rajaonatahina), olivatrakoto@yahoo.fr (O. R. Alson),

andryrasamindrakotroka@hotmail.com (A. Rasamindrakotroka)

${ }^{*}$ Corresponding author

\section{To cite this article:}

Rakotomalala Toky Randriamahazo, Zoliarisoa Ramihajamanana, Anjatiana Annick Raherinaivo, Miora Rasamindrakotroka, Davidra Rajaonatahina, Olivat Rakoto Alson, Andry Rasamindrakotroka. Relationship Between Immunological Factors and Hemarthrosis in Hemophiliacs in Antananarivo Madagascar. International Journal of Immunology. Vol. 7, No. 4, 2019, pp. 47-50.

doi: $10.11648 /$ j.iji.20190704.13

Received: January 14, 2020; Accepted: January 27, 2020; Published: February 13, 2020

\begin{abstract}
Currently, there are 122 hemophiliacs in Madagascar followed at the hemophiliac treatment center of the Joseph Ravoahangy Andrianavalona University Hospital Center (JRA UHC), 55 present hemophilia B and 67 of hemophilia A. In hemophilic patients, the diagnosis of hemarthrosis is obviousin front of articular inflammation. It's important to determinate the main risk factor as well as predisposition indicators tothe occurrence of "spontaneous" hemarthrosis in hemophiliacsfor prevention andearly careanticipation. In this prospect, the search for potentpredisposition indicator such as immunologicalfactorsis important. This is a case control study on all hemophiliacs seen at (JRA UHC) with hemarthrosis for 7 months. We have descriptively studied the qualitative and quantitative variables consisting in the determination of rheumatoid factors (RF) and the titer of antistreptolysin O (ASLO). Then we studied the statistical correlations. During the study period, we included 30 hemophiliac subjects with hemarthrosis who had an average age of 16.8 years. We had as much hemophiliac A as hemophiliac B; $23.3 \%$ practiced sporting activity; $10 \%$ had history of angina, involvement of the knee joint predominated at $44 \%$ (left $24 \%$ ). RF positive were present in $26.7 \%$ (8/30) predominant in hemophiliacs aged from 19 to $36(62.5 \%)$. The ASLO positive titer was found in $43.3 \%(13 / 30)$ predominant in children from 5 to 13 years $(38.5 \%)$ with a maximum rate of $1600 \mathrm{IU} / 1$. There was no significant relationship between the positivity of the parameters with the presence or absence of hemarthrosis with a value of $p=0.231$ and $p=0.06$ respectively $(p>0.05)$. A large number of hemophiliac patients had a combination of clinical and biological signs in relation to diagnose rheumatic fever and rheumatoid arthritis which must be monitored as this could predict the occurrence in the short and medium term of these diseases which could be mistaken for hemarthrosisrelated to hemophilia.
\end{abstract}

Keywords: Hemophilia, Hemarthrosis, Rheumatoid Factor, Antistreptolysin O

\section{Introduction}

Hemophilia is a rare and chronic constitutional hemorrhagic disorder linked to the $\mathrm{X}$ chromosome, transmitted in a recessive mode, hence only occurring in male subjects, encountered in 1/5000 and 1 respectively in
30,000 subjects [1]. Currently, 122 hemophiliacs are registered to Association for the Well Being of Hemophiliacs in Madagascar.

In hemophiliacs patient, hemarthrosisdiagnosis is obviousin front ofarticular inflammation, however many other pathologies such as rheumatoid arthritis or rheumatic 
fever can exhibit the same clinical manifestations. In the objective to search for specific immunological and inflammatory factors that can predispose the occurrence of these hemarthrosis attacks, and to make the diagnosis of rheumatic fever and rheumatoid arthritis in hemophiliacs, aprospective study have been carried out among hemophiliacs seen at JRA UHC with hemarthrosis.

\section{Materials and Methods}

This is a 7-month prospective case-control analytical study from November 2018 to May 2019 carried out at the Hemophilia Treatment Center in Madagascar located at JRA UHC. Our study population consisted of hemophiliacs A and $\mathrm{B}$, enrolled in the register of hemophiliacs members of any age, of any gender, from any geographic and ethnic origin presentinghemarthrosis for the group of case, and the control group showing no hemarthrosis in the three months preceding the study. The study was carried out with the agreement signed by patients or their guardians. For those included in the study, the qualitative variables are constituted by demographic data, history and topography of arthralgia as well as its clinical characteristics (table 1). The quantitative variables are constituted by the results of specific (RF and ASLO) and non-specific (Erythrocyte sedimentation rate, Creactive protein)biological analyzes. (table 2).

Criteria ofJones including Major and Minor Criteria [2], and evidence of prior Group A Streptococcus (GAS) infection allowed diagnosis ofrheumatoid arthritis, and the ACR / EULAR 2009 criteria for the diagnosis of early rheumatoid arthritis [3].

Data were collected on Microsoft office Excel 2013 and the statistical analysis of the data was carried out with $\mathrm{R}$ software. The $\mathrm{p}$ value thresholdis $5 \%$. The chi-square test was used to compare the percentages observed. Student's Ttest was used to compare the means. Measures have been taken for strict confidentiality when preparing the files.

Table 1. Qualitative variables studied.

\begin{tabular}{ll}
\hline QUALITATIVE VARIABLES & PARAMETERS STUDIED \\
\hline Sportingactivities & Yes/ No \\
Types of hemophilia & $\mathrm{A} / \mathrm{B}$ \\
History of trauma & Yes/ No \\
History of angina & Yes/ No \\
History of joint swelling & Yes/ No \\
Types of arthralgia & Monoarthralgia / Polyarthralgia \\
Topography of joint involvement & Shoulder L / R, elbow L / R, wrist L / R, metacarpophalangeal L / R; hip L / R, knee L / R, ankle L / R, \\
Pain schedule & metatarsophalangeal L / R \\
Associated signs & Inflammatory, mechanical, \\
Types of associatedsigns & Yes / No \\
Hemogram & Fever, skin signs, inoculation site, inflammation of the affected joint \\
\hline
\end{tabular}

Table 2. Quantitative variables studied.

\begin{tabular}{ll}
\hline QUANTITATIVE VARIABLES & PARAMETERS STUDIED \\
\hline Rheumatoid factor & $<8$ and $>8 \mathrm{ng} / 1$ \\
Anti-streptolysin O (ASLO) & $\leq 200$ and $>200 \mathrm{UI} / 1$ \\
Erythrocyte sedimentation rate (ESR) & $<10$ and $>10 \mathrm{~mm}$ within 1 hour \\
C-reactiveprotein (CRP) & $<6$ and $>6 \mathrm{mg} / 1$ \\
\hline
\end{tabular}

\section{Results}

There were 12 hemophiliacs registered to ABEHM at the time of the study. During the study period, of the 56 hemophiliacs having presented at least one episode of hemarthrosis, we included 30 patients as cases divided into 16 hemophiliacs A and 14 hemophiliacs B. The average age of the patients was 16.8 years [4 to 36 years]. The number of controls was 8 divided into 4 hemophiliacs $A$ and 4 hemophiliacs B.

Following questionary investigation, $23.3 \%$ of the subjects (7/30) regularly practiced sport. The notion of trauma, even minor, to the joint preceding hemarthrosis in hemophiliacs represented $20 \%(6 / 30)$.. History of recurrent angina was found in $10 \%(3 / 30)$. The history of joint swelling is found in $100 \%$ of cases. Mono articular involvement was present in $100 \%$ of included hemophiliacs with a single topography for
$30 \%$ of cases $(9 / 30)$ versus variable topography for $70 \%$ of cases (21/30). The most common location was the knee joint (44\%) such that for the left, it was $24 \%(7 / 30)$ and for the right $20 \%(6 / 30)$ followed by the right and left elbow (16\%), the right ankle $(10 \%)$ and left $(2 \%)$ and finally the shoulder $(2 \%)$ and the hip. Inflammatory pain predominated in $80 \%$ $(24 / 30)$ of the study population. Associated signs were fever in $16.7 \%(5 / 30)$, inflammatory signs in $96.2 \%(26 / 30)$, bruising in $10 \%(3 / 30)$ and none of the injection sites has been found even in febrile patients.

Rheumatoid factor (RF) was present in $26.7 \%$ or $8 / 30$ hemophiliac subjects with hemarthrosis. With a predominance for subjects in the age group between 19 to 36 years $(62.5 \%)$ with a maximum rate of $64 \mathrm{ng} / 1$. While for the controls, $12.5 \%$ or $1 / 8$ patients had a positive rheumatoid factor level (rate $=16 \mathrm{ng} / \mathrm{l})$. The presence of inflammatory joint pain associated with the positivity of rheumatoid factors was studied and we found no causal relationship between 
them $(\mathrm{p}=0.490)$.

The ASLO titer $>200 \mathrm{IU} / \mathrm{l}$ is present in $43.3 \%$ of hemophiliac subjects with hemarthrosis, that is 13 out of 30 with a predominance in the age group of 5 to 13 years (38.5\%). The maximum titer is 1600 IU / 1 found in an 8year-old child. We found a titer of ASLO raised up to $400 \mathrm{IU}$ / 1 in a control of age group between 5 to 13 years old, associated with joint discomfort.

For the cases, $56.7 \%$ or $17 / 30$ of the subjects had a ESR $>$ $10 \mathrm{~mm}$ in one hour, these ESR varied from 12 to $52 \mathrm{~mm}$ reflecting a non-specific inflammatory phenomenon and $3.3 \%(1 / 30)$ who was a 27 -year-old patient had a CRP of $38.4 \mathrm{mg} / 1$.

Regarding the blood count $36.66 \%$ (11/30) of the cases had a normal blood count and the rest have a slight anemia or neutropenia, no hyperleukocytosis was found

None of the patients were able to meet the Jones' criteria for rheumatic fever or the ACR / EULAR 2009 criteria for the diagnosis of rheumatoid arthritis. All of the control had normal complete blood count, CRP andESR.

The statistical results revealed that there was no relation between the rate of RF or the titer of the ASLO and the localization of the hemarthroses with values of $p>0,05$. The relationship between the presence of a history of recurrent angina and the positivity of ASLO, we do not find any significant link between them with a $\mathrm{p}=0.470$. In the present study, we did not find a relationship between the association of immunological factors and hemarthrosis in hemophiliacs (p: 0.06) (Table 3).

Table 3. Contingency table for immunological factors and hemarthrosis.

\begin{tabular}{lcccc}
\hline & Case $(\mathbf{n}=\mathbf{3 0})$ & Control $(\mathbf{n}=\mathbf{8})$ & $\boldsymbol{p}$ value & Odds ratio \\
\hline Rheumatoid factor & & & & \\
Positive & $8(26.7 \%)$ & $1(12.5 \%)$ & 0.231 & 2.5 \\
Negative & $22(73.3 \%)$ & $7(75.9 \%)$ & & $0.3-24.0$ \\
ASLO & $13(43.3 \%)$ & $1(12.5 \%)$ & 0.06 & 5.2 \\
Positive & $17(56.7 \%)$ & $7(87.5 \%)$ & $0.6-49,1$ \\
Négative & & & \\
\hline
\end{tabular}

\section{Discussion}

The proportion of hemophiliacs with hemarthrosis was low $(46 \%)$ in our study compared to a study by Morillonand al at CHRU of Lille in 2004, they found 75 to $90 \%$ [4]. Frequent incriminated causes are trauma but which was only found in $20 \%$ in our study, similar to the study by Alcalayand al which showed 30\% [5]. The search for other causes favoring these pains seems necessary. Can arthralgia in rheumatic fever and rheumatoid arthritis cause hemarthrosis? Several authors have described that hemarthroses are frequent and characteristic (75-90\% of patients) in hemophiliacs. They are observed at the joints most exposed to trauma: knees, elbows, ankles, hips and shoulders. They arise from learning to walk in severe forms [4]. Mono-articular involvement was present in $100 \%$ of hemophiliacs included. Monoarthritis is characteristic of hemarthrosis, but also sometimes of rheumatic fever as shown in a study by Carapetisand al with a figure of $17 \%(63 / 377)$ [6]. Our young population with an average age of 16.8 years [8-36 years] also confirms this hypothesis. [7]. As for the topography of joint damage, the locations were in order of frequency: the knee joint $44 \%$, the elbow $32 \%$, the ankles $20 \%$ and finally the shoulder and hip $2 \%$ of each reconciling the data from the study by Morillonand al. [5] but also another study carried out in Madagascar on hemophilic arthropathies by Rajaonarisonand al [7]. This obvious polyarthralgia could suspect rheumatoid arthritis or juvenile arthropathy.

The association of inflammatory pain in $80 \%$ (24/30) of the study population and the positivity of rheumatoid factor (26.7\%), and high HSV $(62.5 \%)$ could suspect a preclinical phase rheumatoid arthritis. However, no patient had a sufficient score for the ACR / EULAR 2009 criteria to diagnose rheumatoid arthritis. It is recognized that a high $\mathrm{RF}$ titer in healthy subjects is a predictor of arthritis [3]. The risk of developing RA in a healthy individual is correlated with the RF, the higher the titer, the higher the risk [8]. Numerous studies have pointed out that RF is frequently present in serum several years before clinical manifestations [9]. Individuals with high levels of RF are 26 times more likely to develop RA in the long term, and an absolute 10-year risk of 32\% [10]. The Swedish study by Ärlestigand al. (2011) found a prevalence of $14.0 \%$ in 157 first-degree family members of patients with rheumatoid arthritis with an average age of 54 years (37 to 71 years) [11]. However, no investigation into the family history of MAI and RA has been conducted. The prevalence of autoantibodies in this type of patient increases over time and is highest in the year before the first symptoms. It would therefore be possible to predict the development of rheumatoid arthritis in high-risk populations such as family members who have had several cases of RA, patients with arthralgia and positive serology and to classify them in a target group for early intervention [11]. In these particular cases, the simultaneous search for RF and ACPA appears to be the most specific combination of the prediction of a future disease. Due to lack of means, this exploration have not been carried out in our study.

Second, the frequency of rheumatic fever in hemophiliacs was investigated in our study, but we did not have any significant statistical results. The notion of recurrent angina preceding hemarthrosis, which was one of the signs of acute rheumatic fever was found only in $10 \%$ of cases, the inflammatory signs were presented in $96.2 \%(26 / 30)$ but fever was only present in $16.7 \%(5 / 30)$. The ASLO titer was positive ( $>200 \mathrm{IU} / 1)$ in $43.3 \%$ of hemophiliac subjects with 
hemarthrosisdominatedby children included in the age group of 5 to 13 years $(38.5 \%)$. The maximum titer is $1600 \mathrm{IU} / 1$ found in an 8-year-old child. However, no patient had been able to present a sufficient scorefor the JONES criterion to make the diagnosis of RAA. We found a titer of ASLO raised up to $400 \mathrm{IU} / 1$ in the group age control between 5 to 13 years.

The results were similar to the study by Ben-Chetritand alon a population of 28 subjects $13.8 \%$ of those under 18 had a history of angina, pharyngitis or even tonsillectomy and had a significant positive ASLO in hospitalized patients (33\%) with highertiter rate compared to healthy controls (8\%) [12]. According to the literature, $20 \%$ of group A streptococci do not produce streptolysin $\mathrm{O}$. In addition, some young individuals have an antistreptolysin level between 600 and $1200 \mathrm{IU} / \mathrm{mL}$, without any pathological signification. In this regards, the search of the associationfor the title of antistreptodornase B and anti-streptokinasecould have been helpful but have not been carried out in our study.

\section{Conclusion}

Our study showed that immunological and inflammatory factors do not predispose the onset of these hemarthrosis attacks in hemophiliacs, and none of our patients had sufficient score to diagnose rheumatic fever and rheumatoid arthritis. However, a large number of hemophiliac patients had a combination of clinical and biological signs in relation to these two pathologies which must be monitored as this could predict the occurrence in the short and medium term of these diseases which could be mistaken for hemarthrosisrelated to hemophilia.

\section{Disclosure of Interest}

The authors declare that they have no conflicts of interest concerning this article.

\section{References}

[1] Srivastava, A., Brewer, A. K., Mauser-Bunschoten, E. P., Key, N. S., Kitchen, S., Llinas, A., Street, A. (2013). Guidelines for the management of hemophilia. Haemophilia, 19 (1), e1-e47.

[2] Dajani, A. S., Ayoub, E., Bierman, F. Z., Bisno, A. L., Denny,
F. W., Durack, D. T., \&Karchmer, A. W. (1992). Guidelines for the diagnosis of rheumatic fever: Jones criteria, 1992 update. Jama, 268 (15), 2069-2073.

[3] Peters MJL, Symmons DPM, McCarey D. EULAR evidencebased recommendations for cardiovascular risk management in patients with rheumatoid arthritis and other forms of inflammatory arthritis. Ann Rheum Dis. 2010; 69: 325-31.

[4] D. Morillon, N. Boutry, X. Demondion, B. Duquesnoy, A. Cotten. Lésions musculosquelettiques dans l'hémophilie. EMC-Radiologie. 2004; 1: 283-292.

[5] Alcalay, M., \& Deplas, A. (2002). Prise en charge rhumatologique de l'hémophilie (première partie: manifestations articulaires). Revue du rhumatisme, 69 (9), 868-876.

[6] Martins, Thomas B., Hoffman, James L., Augustine, Nancy $\mathrm{H}$., et al. Comprehensive analysis of antibody responses to streptococcal and tissue antigens in patients with acute rheumatic fever. International immunology, 2008, vol. 20, no 3, p. $445-452$.

[7] Rajaonarison LHNON, FH Rabemanorintsoa, FR Ravelonarivo, OA RakotoAlson, A Ahmad. Profil épidemioclinique et radiologique des atteintes ostéo-articulaires deshémophiles à Madagascar. Pan AfricanMedical Journal. 2014; 19: 287.

[8] Terao C, Yamakawa N, Yano K, Markusse YM, Ikari K, Yoshida $\mathrm{S}$, et al. Rheumatoid Factor Is Associated With the Distribution of Hand Joint Destruction in Rheumatoid Arthritis. Arthritis \& Rheumatology. 2015; 67 (12): 3113-23.

[9] Orge E, Çefle A, Yazıcı A, Gürel-Polat N, Hulagu S. The positivity of rheumatoid factor and anti-cyclic citrullinated peptide antibody in non-arthritic patients with chronic hepatitis C infection. Rheumatol Int. 2010; 30: 485-8.

[10] Deane KD, Norris JM, Holers VM. Preclinical rheumatoid arthritis: identification, evaluation, and future directions for investigation. Rheum Dis Clin North Am. 2010; 36 (2): 21341.

[11] Remus CR, Castillo-Ortiz JD, Aguilar-Lozano L, PadillaIbarra J, Sandoval-Castro C, Vargas-Serafin CO, et al. Autoantibodies in Prediction of the Development of Rheumatoid Arthritis Among Healthy Relatives of Patients With the Disease. Arthritis Rheum. 2015; 67 (11): 2837-44.

[12] Ben-chetrit E, Moses AE, Agmon-levin N, Block C. Serum levels of antistreptolysin $\mathrm{O}$ antibodies : their role in evaluating rheumatic diseases. International journal of rheumatic diseases. 2012; 15 (1): 78-85. 